\title{
Variability in Delivered Dose from Pressurized Metered-Dose Inhaler Formulations Due to a Delay Between Shake and Fire
}

\author{
Ross H.M. Hatley, PhD, Jacob Parker, BSc, John N. Pritchard, PhD, and Dirk von Hollen, BSc ${ }^{2}$
}

\begin{abstract}
Background: Pressurized metered-dose inhalers (pMDIs) should be shaken before use to prevent creaming or sedimentation of the drugs in solution; however, data published on this topic are limited, and it is rarely specified how soon after shaking the device should be actuated. Delays between shaking and firing the pMDI have previously been shown to cause significant inhomogeneity in delivered dose. We studied the effect of various shake-fire delays on the drug delivered from five commercially available pMDIs commonly prescribed for asthma and chronic obstructive pulmonary disease to assess the potential variability in delivered dose.

Methods: The pMDI formulations tested were the Flovent HFA, Ventolin Evohaler, Airomir Inhaler, and Symbicort (suspension pMDIs), and the QVAR 100 Inhaler (solution pMDI). Each pMDI was shaken for 5 seconds before attachment to a dosage unit sampling apparatus collection tube and filter, and it was actuated once with shake-fire delays of $0,5,10,20,30,40,50$, and 60 seconds. Analysis of the eluates from the collection tubes and filters was performed by using high-performance liquid chromatography. Three of each pMDI were tested twice with each time delay.

Results: All of the suspension pMDIs produced variable amounts of drug over the shake-fire delays tested. A comparison of the delivered doses after the 0-and 60-second delays showed that the drug delivered increased for the Flovent HFA (320\%), Ventolin Evohaler (346\%), and Airomir Inhaler (230\%) pMDIs; decreased for the Symbicort budesonide (75\%) and formoterol fumarate (76\%) pMDI; and remained consistent for the QVAR 100 Inhaler pMDI. Conclusions: The amount of drug delivered can vary widely over different shake-fire delays with suspension pMDIs. Therefore, guidance should be given to users/caregivers on the timing of firing after shaking their device, particularly with pediatrics, who may take time to become receptive to accepting their medication after pMDI shaking and before dose administration.
\end{abstract}

Keywords: creaming, delivered drug, formulation, pressurized metered-dose inhaler (pMDI), sedimentation, shake-fire delay

\section{Introduction}

$\mathbf{T}$ HE PRESSURIZED METERED-DOSE INHALER (pMDI) has been in use since 1956 to deliver aerosolized medications to patients with respiratory conditions, most commonly asthma and chronic obstructive pulmonary disease, and it is currently the most commonly used device for aerosolized drug delivery worldwide. ${ }^{(1,2)}$ Advances in pMDI design and the production of add-on devices, such as valved holding chambers (VHCs) and facemasks, have expanded the range of patients who are treatable by pMDI aerosol drug delivery to include those who are unable to coordinate their inhalation with device actuation, such as the very young.

Historically, pMDI formulations included chlorofluorocarbon (CFC) propellants to produce the aerosolized drug, but in 1987, the Montreal Protocol ruled that CFCs were to be banned due to their detrimental effects on the ozone layer; therefore, alternatives to CFC propellants had to be found.

\footnotetext{
${ }^{1}$ Philips Respironics Respiratory Drug Delivery (UK) Ltd, a business of Philips Electronics UK Limited, Chichester, United Kingdom.

${ }^{2}$ Respironics, Inc., A Philips Healthcare Company, Murrysville, Pennsylvania.

(c) Ross H.M. Hatley, et al., 2017. Published by Mary Ann Liebert, Inc. This Open Access article is distributed under the terms of the Creative Commons Attribution Noncommercial License (http://creativecommons.org/licenses/by-nc/4.0) which permits any noncommercial use, distribution, and reproduction in any medium, provided the original author(s) and the source are credited.
} 
Hydrofluoroalkanes (HFAs) were found to be the most appropriate alternative to CFCs; however, the transfer from CFC- to HFA-based formulations was not straightforward. Issues, such as the differences in physicochemical properties between the different HFA formulations, the practical difficulties of working with a propellant that is a gas at room temperature, and finding surfactants that are soluble in HFA formulations, delayed the process. ${ }^{(3)}$

In most countries, the transition from CFC to HFA pMDI propellants has been completed, but differences in the physicochemical properties of these propellants, such as polarity and density, have resulted in changes to the design of pMDIs (such as to the valves and elastomers) and differences in formulation approaches (such as no excipient, addition of ethanol or glycerol, or particle engineering). ${ }^{(3)}$

Differences in the replacement formulations also exist; both HFA-227 and HFA-134a were selected for use in pMDI devices as they most closely matched the properties of the previously used CFCs, but these formulations differ slightly in boiling point, vapor pressure, density, and water solubility. ${ }^{(3,4)}$ There are, therefore, numerous variations in the formulations and designs of HFA pMDIs, even for delivery of the same active ingredient, although these differences can allow for the most appropriate characteristics of the HFA formulations to be matched with the particular requirements of the active drug to be delivered.

The widespread use of pMDIs makes it essential to ensure that all patients are able to use their device correctly to achieve an adequate dose and to maintain disease control. However, this is often not the case; patient compliance with the pMDI technique has previously been found to be poor, ${ }^{(5,6)}$ and poor technique has even been observed in healthcare providers. ${ }^{(7-10)}$

Correct use of a pMDI requires the performance of a number of key steps to achieve effective drug delivery [Table $1^{(11)}$; these steps are generally the same between different pMDIs due to the similar device designs and operating principles. For suspension formulations, shaking the pMDI is an essential step to ensure that the aerosol released from the device contains a uniform drug dose. ${ }^{(12)}$ As most patients and doctors would not know whether the drug is in

Table 1. Metered-Dose Inhaler Technique

\begin{tabular}{cl}
\hline Step & \multicolumn{1}{c}{ Instruction } \\
\hline 1 & Take the cap off. \\
2 & Shake the inhaler. \\
3 & Seal lips around mouthpiece while opening the teeth. \\
4 & Start breathing in slowly through the mouth. \\
5 & Then, activate inhaler while continuing to breathe in. \\
6 & Breathe in slowly, over at least 5 seconds until \\
& lungs are full. \\
7 & $\begin{array}{l}\text { Hold your breath as long as you comfortably can, } \\
\text { then relax. }\end{array}$ \\
8 & $\begin{array}{l}\text { Replace the cap. } \\
9\end{array}$ \\
& $\begin{array}{l}\text { Check the dose counter on your inhaler regularly } \\
\text { (if it has one) so you know when to get a new }\end{array}$ \\
10 & Ane in good time.
\end{tabular}

Reproduced from The Aerosol Drug Management Improvement Team $^{(11)}$ (www.admit-online.info/en/). solution or suspension, it has become a universal instruction to shake any pMDI before use, but despite this, many patients still do not adequately shake their pMDI before use. ${ }^{(13)}$

The timing between shaking the pMDI and actuating a dose is rarely specified in the patient instruction leaflet of the prescribed pMDI, and may be an important area of research, as it has already been shown that suspension formulations within an HFA pMDI can cream or sediment soon after the device has been shaken ${ }^{(14-17)}$ due to density differences between the drug and the propellant, ${ }^{(15)}$ and this has also been observed in a clinical setting. ${ }^{(17)}$

In devices in which creaming occurs, the drug particles float to the top of the pMDI canister, as they are less dense than the propellants in the solution. If the amount of time taken to shake the pMDI and the intensity of this shake are not sufficient to achieve a uniform dispersion of the drug in liquid, or if there is a delay between shaking and firing, there is a risk that on firing, the retaining cup ${ }^{(18)}$ would re-fill with a formulation containing a lower concentration of drug, leading to a reduced concentration of drug available for entry into the metering valve on next use. As a good mix between the content of the valve metering chamber and the bulk formulation is difficult to achieve, it is likely that the next dose would be lower than expected.

Over time, repeated actuations of these lower doses would result in a more concentrated drug formulation in the remaining solution within the canister; therefore, toward the end of the canister life, the drug concentration within the canister would be much higher than the label claim, resulting in higher drug deliveries to the patient. ${ }^{(15,19)}$ The opposite occurs during drug sedimentation; after shaking, the drug particles sink to the bottom of the pMDI canister as they are denser than the surrounding propellants, resulting in a higher concentration of drug to the patient in the shots at the start of the canister life, and a decreasing drug concentration per shot toward the end of the canister life.(20)

Data available on the subject of creaming and sedimentation of HFA pMDI drug formulations currently in use by patients are limited, especially with respect to the acceptable length of time between shaking the device and actuating a shot. Simple mistakes in real-life pMDI use, such as dropping the pMDI, being distracted by a telephone, and difficulties in attaching the pMDI to a VHC, can increase the time between shaking the device and actuating a shot. The common nature of mistakes that cause shake-fire delays and general poor compliance with the pMDI technique among patients ${ }^{(5,6)}$ indicate that this is an important topic.

The purpose of this study was to investigate the effects of various lengths of shake-fire delay on the drug delivered by currently available HFA suspension pMDIs, and an HFA solution pMDI to act as a control, as solution pMDIs are homogenous and do not exhibit sedimentation. Five different pMDIs were tested over a range of shake-fire delay times. The formulations of the pMDIs used included suspensions without excipients, suspensions with excipients, and solutions.

\section{Materials and Methods}

\section{Test products}

Five HFA pMDI brands were tested (Table 2), and three of each pMDI brand were analyzed during the study. The 
Table 2. The pMDi Drug Formulations Tested

\begin{tabular}{|c|c|c|c|c|c|c|c|}
\hline$p M D I$ & Drug and label claim & $\begin{array}{l}\text { Drug } \\
\text { density } \\
(g / m L)\end{array}$ & $\begin{array}{l}\text { Pack size } \\
\text { (no. of } \\
\text { puffs) }\end{array}$ & Propellant & $\begin{array}{l}\text { Propellant } \\
\text { density } \\
(\mathrm{g} / \mathrm{mL})\end{array}$ & $\begin{array}{l}\text { Drug/ } \\
\text { propellant } \\
\text { density } \\
\text { difference } \\
(\mathrm{g} / \mathrm{mL})\end{array}$ & $\begin{array}{c}\text { Formulation } \\
\text { type }\end{array}$ \\
\hline $\begin{array}{l}\text { Flovent HFA } \\
\text { (GlaxoSmithKline, } \\
\text { Research Triangle } \\
\text { Park, NC) }\end{array}$ & $\begin{array}{l}220 \mu \mathrm{g} \text { fluticasone } \\
\text { propionate }\end{array}$ & 1.33 & 120 & HFA-134a & 1.22 & 0.11 & Suspension \\
\hline $\begin{array}{l}\text { Ventolin Evohaler } \\
\text { (GlaxoSmithKline } \\
\text { UK, Uxbridge, } \\
\text { UK) }\end{array}$ & $\begin{array}{l}100 \mu \mathrm{g} \text { salbutamol } \\
\text { sulfate }\end{array}$ & 1.30 & 200 & HFA-134a & 1.22 & 0.08 & Suspension \\
\hline $\begin{array}{l}\text { Airomir Inhaler } \\
\text { (TEVA UK Ltd, } \\
\text { Harlow, UK) }\end{array}$ & $\begin{array}{l}100 \mu \mathrm{g} \text { salbutamol } \\
\text { sulfate }\end{array}$ & 1.30 & 200 & HFA-134a & 1.22 & 0.08 & $\begin{array}{l}\text { Suspension and } \\
\text { excipient } \\
\text { (ethanol and } \\
\text { oleic acid) }\end{array}$ \\
\hline $\begin{array}{l}\text { Symbicort } \\
\text { (AstraZeneca, } \\
\text { Wilmington, DE) }\end{array}$ & $\begin{array}{l}160 \mu \mathrm{g} \text { budesonide } \\
4.5 \mu \mathrm{g} \text { formoterol } \\
\text { fumarate }\end{array}$ & $\begin{array}{l}1.30 \\
1.24\end{array}$ & 120 & HFA-227 & 1.41 & $\begin{array}{l}-0.11 \\
-0.17\end{array}$ & $\begin{array}{l}\text { Suspension and } \\
\text { excipients } \\
\text { (povidone K25 } \\
\text { USP and } \\
\text { polyethylene } \\
\text { glycol) }\end{array}$ \\
\hline $\begin{array}{l}\text { QVAR } 100 \text { Inhaler } \\
\text { (TEVA UK Ltd) }\end{array}$ & $\begin{array}{l}100 \mu \mathrm{g} \text { beclomethasone } \\
\text { dipropionate }\end{array}$ & $\begin{array}{c}1.26 \\
\text { (solution) }\end{array}$ & 200 & HFA-134a & 1.22 & N/A & Solution \\
\hline
\end{tabular}

HFA, hydrofluoroalkane; pMDI, pressurized metered-dose inhaler; USP, United States Pharmacopeia.

devices were new, unused pMDIs and the order of testing was to use shake-fire delays of $0,5,10,20,30,40,50$, and 60 seconds, followed by a repeat test with the same canister. The pMDIs were primed by firing three shots to waste before each test and repeat test, and no shots were fired to waste during the tests. The ambient conditions within the laboratory during testing were $21.6^{\circ} \mathrm{C}-22.6^{\circ} \mathrm{C}$ air temperature, 1011-1022 mbar air pressure, and 50.0\%-59.2\% relative humidity.

\section{Test plan}

A pMDI holds a single shot of drug in the metering chamber, ${ }^{(18)}$ and it is this drug that is delivered when the device is actuated. This means that the shot administered for the "current" actuation is a product of the propellant and drug formulation filled into the metering chamber at the end of the last actuation. Consequently, there is a one-shot delay between shaking the device and the delivery of the shot corresponding to that shake ${ }^{(18)}$ (Fig. 1). Therefore, this delay in the release of the dose associated with the shake-fire delay was accounted for by reassigning the previous shake-fire delay to the correct filter, to associate the appropriate delay with the shot collected (Fig. 2). The device was not primed between the different shake-fire delays to preserve the one-shot delay system (Fig. 2).

\section{Experimental procedure}

An HCP5 vacuum pump (Copley Scientific Ltd, Nottingham, UK) was attached to a filter support base, which was mounted on a stand by using a clamp. A dosage unit sampling apparatus (DUSA; Copley Scientific Ltd) collection tube and a DUSA filter were added to the filter support base, and an end connector was attached to the other end of the DUSA. A TSI flow meter (TSI Incorporated, Shoreview, MN) was attached to the end connector with connectors and tubing, and the flow was set to $30 \mathrm{~L} / \mathrm{min}$, as stated in the United States Pharmacopeia (USP). ${ }^{(21)}$ Once the flow had been set, the TSI flow meter and the end connector were removed and a metered-dose inhaler (MDI) custom connector for DUSA was attached in their place.

Each pMDI (Table 2) was shaken for 5 seconds, as stated in the USP ${ }^{(21)}$ before being connected to the DUSA via the MDI custom connector for DUSA. If it was not possible to attach the pMDI to the custom connector without agitating the canister, the plastic actuator with the canister removed was attached to the connector first; then, the canister was shaken separately before being placed carefully back into the actuator before firing. After being shaken and attached to the DUSA, the pMDI was actuated and held down for 1-2 seconds, and the pump was stopped after $\sim 10$ seconds. The pMDI and MDI custom connector for DUSA were removed, and a DUSA end cap was placed on the open end of the collection tube. The DUSA was removed from the vacuum connector, and a check was completed to ensure that the filter was inside the connection tube.

The appropriate diluent was added to the collection chamber, and a second end cap was connected to the open end of the DUSA. The DUSA was then shaken vigorously to wash all of the inside surfaces fully. An aliquot of the liquid was collected via a plastic syringe from the DUSA and was filtered through a $30 \mathrm{~mm}, 0.2 \mu \mathrm{m}$ nylon syringe into a highperformance liquid chromatography (HPLC) vial for analysis. For the Symbicort inhaler, two aliquots were taken from each DUSA during each test and were transferred to separate HPLC vials. Of these two Symbicort samples, one was tested for budesonide and the other was tested for formoterol fumarate. 
Suspension evenly distributed in propellant

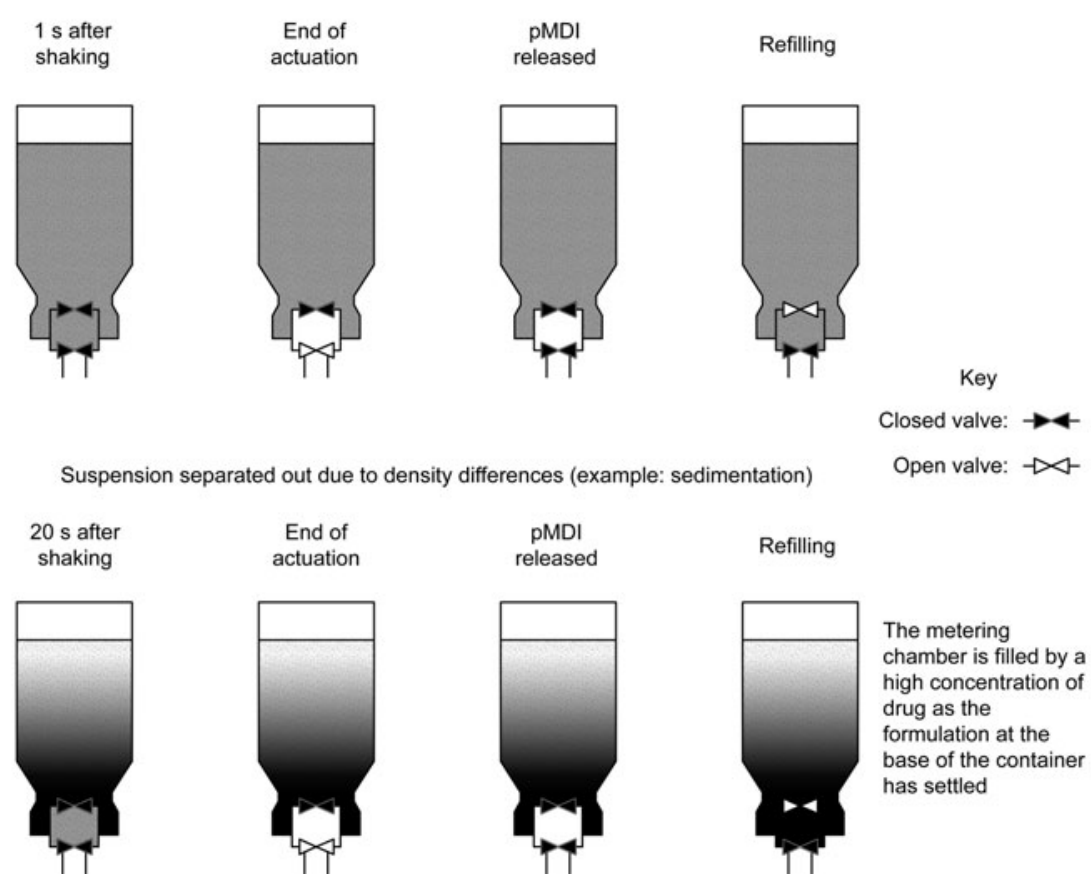

FIG. 1. A representation of the 1-shot delay between the shaking of the device and the corresponding shot of drug.

\section{HPLC analysis}

The HPLC system (Agilent 1100 Series; Agilent Technologies, Waldbronn, Germany) was fitted with the appropriate column, depending on the drug under test (fluticasone propionate: Waters Symmetry C18 column [ $5 \mu \mathrm{m}, 250 \times 4.6 \mathrm{~mm}, 40^{\circ} \mathrm{C}, 1.5 \mathrm{~mL} / \mathrm{min}$; Waters Corporation, Milford, MA]; salbutamol sulfate: Genesis Phenyl column $\left[4 \mu \mathrm{m}, 150 \times 4.6 \mathrm{~mm}, 22^{\circ} \mathrm{C}, 1 \mathrm{~mL} / \mathrm{min}\right.$; Crawford Scientific, Lanarkshire, UK]; budesonide: Supelcosil LC-18 column [ $5 \mu \mathrm{m}, 50 \times 4.6 \mathrm{~mm}, 22^{\circ} \mathrm{C}, 2 \mathrm{~mL} / \mathrm{min}$; Sigma-Aldrich, St. Louis, MO]; formoterol fumarate: Waters Spherisorb ODS 2 column $\left[3 \mu \mathrm{m}, 125 \times 4.6 \mathrm{~mm}, 30^{\circ} \mathrm{C}, 1.5 \mathrm{~mL} / \mathrm{min}\right.$; Waters Corporation]; beclomethasone dipropionate: Phenomenex Luna C18(2) column $\left[3 \mu \mathrm{m}, 150 \times 4.6 \mathrm{~mm}, 25^{\circ} \mathrm{C}, 1 \mathrm{~mL} / \mathrm{min}\right.$; Phenomenex, Inc., Torrance, CA]).

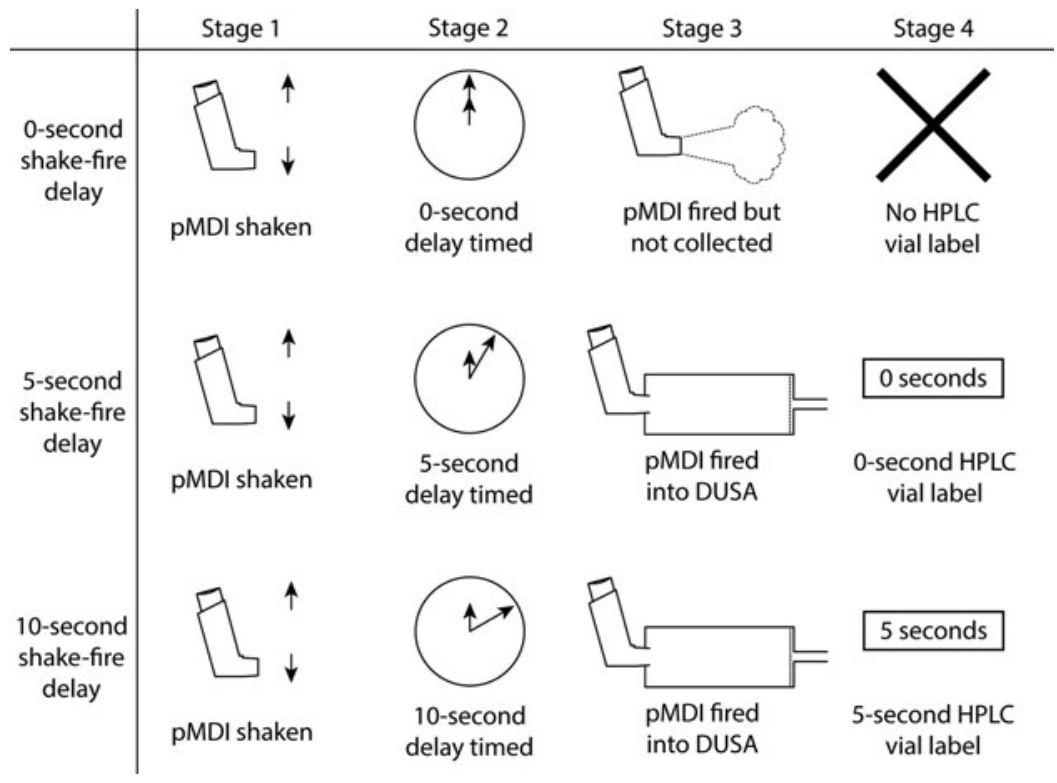

FIG. 2. A schematic of the method used to compensate for the 1-shot delay with the 0-, 5-, and 10-second shake-fire delays shown as examples. 
Bracketing standards of each of the drugs were made and run, and the accuracy of the standards was checked. System suitability was then checked by using five replicate injections of the bracketing standards. The relative standard deviations had to be either at or below $2 \%$, and the USP tailing factor of the drug peak had to be no greater than the predetermined values (fluticasone propionate, budesonide, and beclomethasone dipropionate: 1.5, salbutamol sulfate and formoterol fumarate: 2.0 ) for the results of the run to be considered valid.

\section{Results}

There were three different profiles of drug delivery related to increasing shake-fire delay times; these were profiles of increased drug delivery, reduced drug delivery, and drug delivery unaffected by shake-fire delay time. The increased and reduced drug delivery profiles corresponded with the pMDIs with suspension formulations, and the unaffected profile corresponded with the pMDI with a solution formulation (Fig. 3).

Results for the Flovent HFA, Ventolin Evohaler, and Airomir Inhaler pMDIs exhibited an increase in the mass of drug delivered. When compared with the 0 -second shake-fire delay, this increase resulted in a doubling of the amount of drug delivered after a 20 -second shake-fire delay for the Flovent HFA and Ventolin Evohaler pMDIs, and after a 50second shake-fire delay for the Airomir Inhaler pMDI (Fig. 4). The increase in delivered drug appeared to plateau at around the 60-second shake-fire delay for the Flovent HFA and Ventolin Evohaler pMDIs. However, there was no plateau observed with the Airomir Inhaler, as the increase in delivered drug over the range of shake-fire delays was more gradual and the rate of increase was fairly consistent. The mass of drug delivered by the Flovent HFA, Ventolin Evohaler, and Airomir Inhaler pMDIs after a 60-second shake-fire
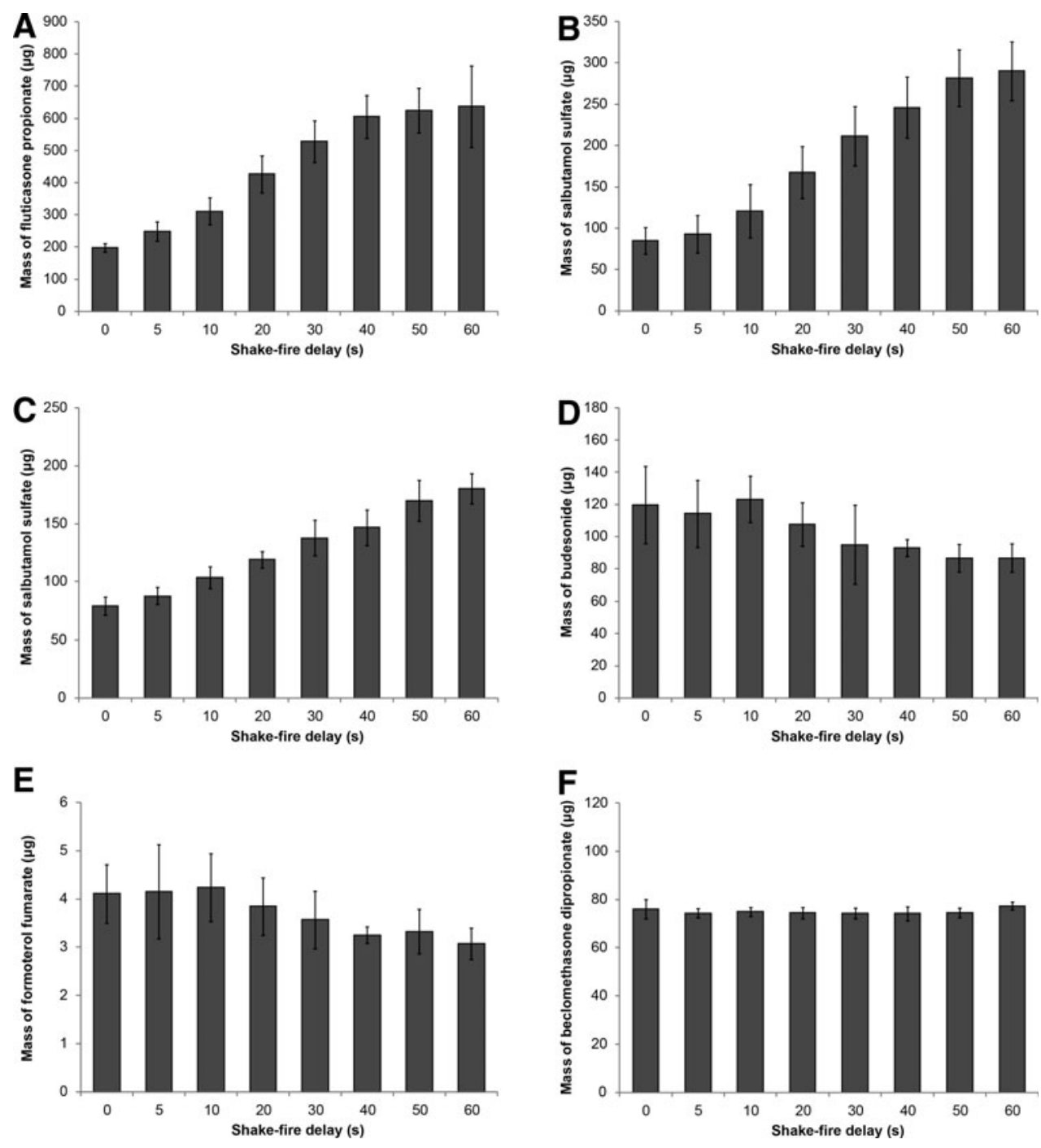

FIG. 3. Mean mass of drug delivered from the (A) Flovent HFA, (B) Ventolin Evohaler, (C) Airomir Inhaler, Symbicort [(D) budesonide and (E) formoterol fumarate], and (F) QVAR 100 Inhaler pMDIs across the range of shake-fire delays. The error bars denote 1 standard deviation about the mean $(n=6)$. HFA, hydrofluoroalkane; pMDI, pressurized metered-dose inhaler. 


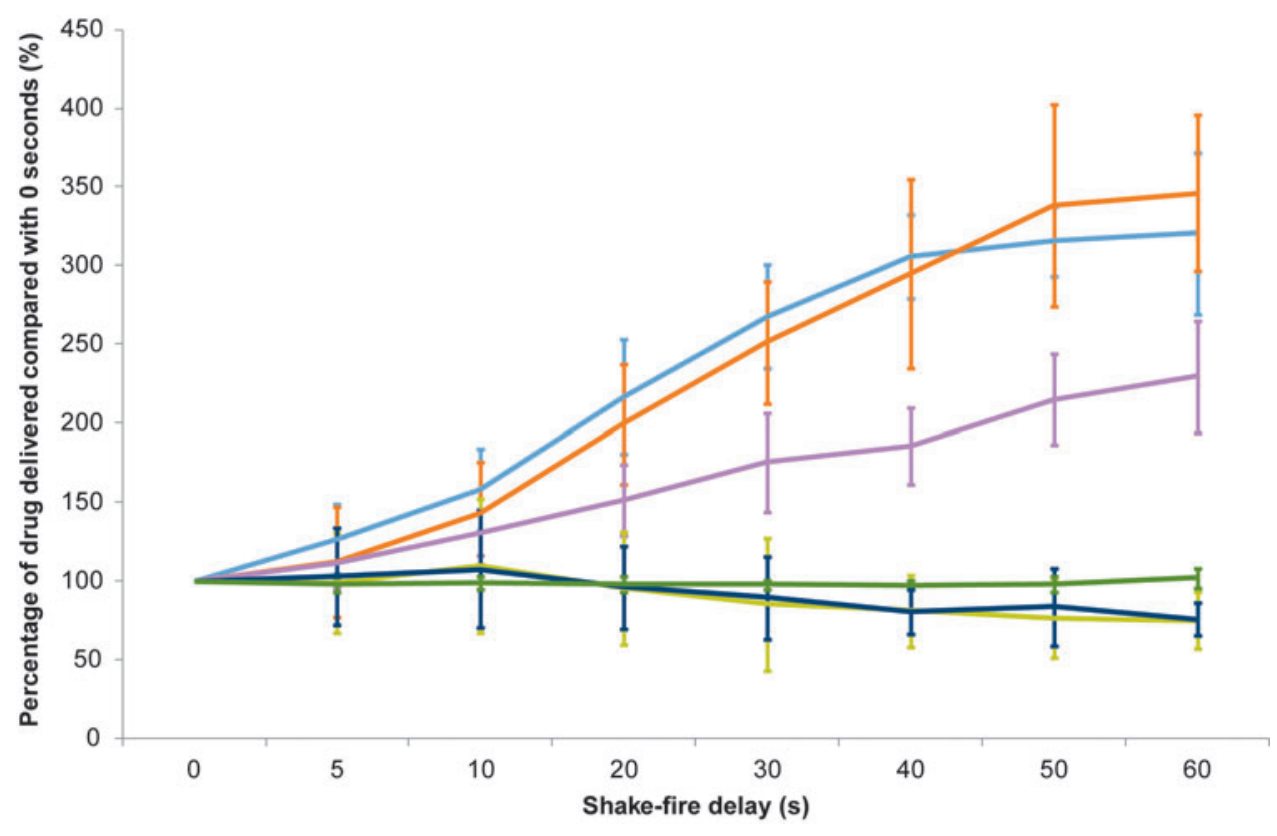

FIG. 4. Mean percentage of drug delivered across the range of shake-fire delays compared with the drug delivered at 0 seconds by the Flovent HFA ( $)$, Ventolin Evohaler ( $)$, Airomir Inhaler ( ), Symbicort (budesonide [ ] and formoterol fumarate [ ] ]), and QVAR 100 Inhaler $(\square)$ pMDIs. The error bars denote 1 standard deviation about the mean $(n=6)$.

delay was $320 \%, 346 \%$, and $230 \%$ of the drug delivered with the 0 -second shake-fire delay, respectively (Fig. 4).

Conversely, the results for the Symbicort pMDI, which comprises a formulation of two drugs (budesonide and formoterol fumarate), exhibited a reduction in drug delivered over increasing shake-fire delay times between 20 and 60 seconds. There was also a close similarity in the delivery profiles of the two drugs, as shown in Figure 4. The effect of shake-fire delay time on the amount of drug delivered was lower for the Symbicort drugs compared with the other suspension-formulated pMDIs, with around $75 \%$ of the 0 -second dose delivered after 60 seconds for both the drugs.

Finally, results for the QVAR 100 Inhaler showed that the amount of drug delivered from this pMDI was relatively unaffected by shake-fire delay time, with consistent amounts of drug delivered across all the shake-fire delay times tested.

\section{Discussion}

These results show a wide variation in the effect of delays between shaking and firing on the dose delivered from pMDIs commonly used for the treatment of respiratory conditions. The reason for this variation lies in the different approaches to pMDI formulation used in the different brands of pMDI.

The large increases in drug delivered by the Flovent HFA, Ventolin Evohaler, and Airomir Inhaler suspension pMDIs as time between shaking and actuating the device increased (Fig. 3) were likely due to sedimentation of the drug particles within the canister, ${ }^{(14,20)}$ which occurs when the drug particles are denser than the propellant. ${ }^{(20)}$ All three of these formulations are based on the HFA-134a propellant, which has a density of $1.22 \mathrm{~g} / \mathrm{mL}$, whereas the density of fluticasone propionate is $1.33 \mathrm{~g} / \mathrm{mL}$, and that of salbutamol sulfate is $1.30 \mathrm{~g} / \mathrm{mL}$ (Table 2). This difference in densities is likely to be the cause of the sedimentation observed, as the drug particles are denser than the propellant. ${ }^{(15)}$ The rapid increases in delivered drug observed between the 10- and 40second shake-fire delays for the Flovent HFA pMDI, and the 10- and 50-second shake-fire delays for the Ventolin Evohaler pMDI (Fig. 3) may correspond to the sedimentation of larger particles, as their greater mass overcame the resistance of the solvent to particle movement.

After the initial rapid increases in delivered drug produced by the Flovent HFA and Ventolin Evohaler pMDIs, the delivered drug appeared to plateau at a level that was more than threefold higher than the drug delivered with the 0 -second shake-fire delay. This suggests that a significant further increase in delivered drug is unlikely to occur after a shake-fire delay of $\sim 50$ seconds, as most of the larger drug particles will have already sedimented by this time, and the smaller particles are likely to remain suspended for longer.

The drug delivered by the Airomir Inhaler pMDI increased more gradually over the shake-fire delay times tested and did not level off after a certain time point; therefore, it may be expected to increase further with shakefire delays that are longer than 60 seconds. As the differences between the drug and propellant densities were the same for the Airomir Inhaler and the Ventolin Evohaler pMDIs, the more gradual sedimentation observed is unlikely to be due to the difference between the drug and propellant densities. Instead, this more gradual sedimentation may be the result of the ethanol-induced dissolution of oleic acid, which acts as a surfactant in the formulation. ${ }^{(4)}$ Therefore, suspension formulations with added ethanol excipients could be less prone to deviations from the expected dose arising from short shake-fire delays; however, a similar level 
of sedimentation could occur over longer periods. Suspensions with smaller differences between drug and propellant densities and added ethanol excipients may be more robust against delays between shaking and firing, as the increase in delivered drug with a 60-second shake-fire delay was approximately equivalent to that with a 20 - to 30 -second shake-fire delay for products without surfactants (Flovent HFA and Ventolin Evohaler; Fig. 4).

The Symbicort formulation remained much more stable over the period of shake-fire delays tested, with a reduction in the amount of drug delivered after a delay of around 20 seconds, which indicates that creaming of the formulation had occurred. In this formulation, HFA-227 is used, which has a density of $1.41 \mathrm{~g} / \mathrm{mL}$, which is higher than the density of $1.30 \mathrm{~g} / \mathrm{mL}$ for budesonide and of $1.24 \mathrm{~g} / \mathrm{mL}$ for formoterol fumarate (Table 2). Creaming rather than sedimentation would, therefore, be expected, as the drug is less dense than the propellant.

The decrease in delivered drug from the Symbicort pMDI was similar for both budesonide and formoterol fumarate $(75 \%$ and $76 \%$ of the drug delivered with the 0 -second shake-fire delays was delivered with the 60-second shake-fire delays, respectively), and the trends of both drugs over the time delays tested almost exactly matched, suggesting that the individual budesonide and formoterol fumarate particles may have been weakly bonded to one another. ${ }^{(22)}$ These slight decreases in delivered drug were proportionally far smaller than the increases observed with the other suspension pMDIs, despite the differences between drug and propellant densities being the largest of the pMDIs tested (Table 2). This was likely a result of the added excipients slowing the creaming process, as with the excipients that slowed the process of sedimentation in the Airomir Inhaler pMDI.

The results for the QVAR 100 Inhaler were the most stable of the pMDIs tested, with a consistent amount of drug delivered over all of the shake-fire delays, and no trend of increasing or decreasing delivered dose over the period of shake-fire delays tested. As the drug within the QVAR 100 Inhaler pMDI is in a solution formulation, it would be expected that shaking the device would have no impact on the delivered mass of the drug. This is because the drug in a solution formulation is in a dissolved state within the liquid propellant, instead of small drug particles suspended in liquid (as in a suspension formulation), and would, therefore, be unable to sediment or cream. The consistency in dosing observed in the current study, therefore, supports the expectation that there would be no effect of a shake-fire delay on the solution formulation of the QVAR 100 Inhaler pMDI.

The patient information leaflets provided with pMDIs state that the device must be shaken before use (with the exception of the QVAR 100 Inhaler solution pMDI); however, it is rarely specified how soon before use the device should be shaken. In light of the results of this study, it is clear that any delay between shaking and actuating the pMDI may have a substantial effect on the amount of drug delivered.

In previous studies, it has been shown that around $23 \%-$ $50 \%$ of patients do not shake their device before actuation, ${ }^{(23,24)}$ and for those who do, there can be a wide range in the number of seconds between shaking the device and actuating a shot. ${ }^{(24)}$ Such delays can occur when trying to administer a dose through a VHC to a child, or when adult patients drop the device or are distracted by external factors; furthermore, a study by Berlinski et al. has shown that delays between shaking and firing a pMDI can cause variability in the delivered dose in a clinical setting. ${ }^{(17)}$ The results of this study indicate that even small delays of 5-10 seconds are enough to increase the mass of drug delivered to above the nominal dose in some suspension pMDIs. This variability in the amount of drug delivered could potentially lead to delivered doses far in excess of the dose intended by the prescribing physician. Systematic use with delays of more than 20 seconds during the early shot-life of the pMDI could lead to prolonged over-exposure and the potential for adverse effects such as oral candidiasis and pharyngitis. ${ }^{(25,26)}$ Furthermore, if excessive doses were to be delivered consistently, this would deplete the amount of bulk drug within the pMDI by a greater amount than expected over time, and it could lead to systematic under-dosing later in the shot-life of the pMDI, which could have implications for the clinical efficacy of the treatment and control of the disease being treated.

The purpose of this study was to investigate the effect of shake-fire delays on delivered dose and, as such, we used the first 21 shots from a new pMDI to avoid the secondary effects of drug concentration changes in the drug left in the pMDI across use. However, we did not address the effects of shake-fire delays on drug delivery toward the end of the canister life, nor did we randomize the shake-fire delays. Further investigation using the same shake-fire delays across the complete life of a canister would be useful to further understand the variability in drug delivery from pMDIs.

It has been shown that patients often do not shake their pMDI before use, ${ }^{(23,24)}$ and in studies with CFC pMDIs, not shaking for a minimum of 16 hours before actuation resulted in $25.5 \%$ reductions in the amount of drug delivered. ${ }^{(12)}$ Given the very large changes in delivered drug observed in this study with a shake-fire delay of just 60 seconds, it could be beneficial to investigate the drug-release profile across the container life to evaluate the amount of drug that would be received by patients from an HFA pMDI if it was not shaken before use.

Laboratory and regulatory tests of the uniformity of dose from pMDIs are performed under strictly controlled conditions to ensure that reproducible doses of medicinal drug are delivered when the device is used according to the manufacturer's instructions. However, none of the package inserts for the pMDIs tested specify the allowable time between shaking and firing the pMDI. The very wide variation in delivered dose after only a short period of delay suggests that specification of the time between shaking and firing the pMDI is a critical parameter in reducing the likelihood of administration of a dose that is widely different to that prescribed by the clinician. In a recent in vivo study, we observed emitted doses of $222 \%$ of the nominal dose after a 30 -second delay during treatment of children using a pMDI. ${ }^{(17)}$ Delays of this magnitude could occur when attempting to administer aerosol treatment to young children with a pMDI and VHC.

\section{Conclusions}

The results from this study have shown that drug formulations from suspension pMDIs can begin to cream or 
sediment immediately after shaking the pMDI, whereas solution formulations appear to remain stable over a range of shake-fire delays. The effects vary across different formulations and are less severe in pMDI formulations with added excipients, but even with excipients, the amount of drug delivered can vary widely with long shake-fire delays. It is, therefore, important to ensure that patients who use pMDIs are given guidance to actuate a dose as soon as possible after shaking their device to reduce the likelihood of variation in delivered drug dose, particularly caregivers who administer doses to pediatric patients, who may take some time to settle after the shaking of the pMDI, before administration of the dose.

\section{Acknowledgments}

The authors acknowledge Rachael Giles for drafting assistance and Sophia Kuperman for editorial assistance (PS5 Consultants Ltd, Portsmouth, Hampshire, UK).

\section{Author Disclosure Statement}

R.H.M.H., J.P., and J.N.P. are employees of Respironics Respiratory Drug Delivery (UK) Ltd, a business of Philips Electronics UK Limited, Chichester, West Sussex, UK. D.v.H. is an employee of Respironics, Inc., a Philips Healthcare company, Murrysville, PA.

\section{References}

1. Lavorini F, Corrigan CJ, Barnes PJ, Dekhuijzen PRN, Levy ML, Pedersen S, Roche N, Vincken W, and Crompton GK: ADMIT: Retail sales of inhalation devices in European countries: So much for a global policy. Respir Med. 2011; 105:1099-1103.

2. Atkins PJ, Woodcock A, Blinova O, Khan J, Stechert R, Wright P, and Yizhong Y: Chapter 8-Medical aerosols. In: Intergovernmental Panel on Climate Change (IPCC), (ed). Safeguarding the Ozone Layer and the Global Climate System: Issues Related to Hydrofluorocarbons and Perfluorocarbons. Cambridge University Press, Cambridge; pp. 349-360, 2005.

3. Pritchard JN: Recent advances in drug delivery via pressured metered-dose inhalers. In: L Gradoń and J Marijnissen, (eds). Optimization of Aerosol Drug Delivery. Kluwer Academic Publishers, The Netherlands; pp. 105121, 2003.

4. Myrdal PB, Sheth P, and Stein SW: Advances in metered dose inhaler technology: Formulation development. AAPS PharmSciTech. 2014;15:434-455.

5. Crompton GK, Barnes PJ, Broeders M, Corrigan C, Corbetta L, Dekhuijzen R, Dubus JC, Magnan A, Massone F, Sanchis J, Viejo JL, and Voshaar T; Aerosol Drug Management Improvement Team: The need to improve inhalation technique in Europe: A report from the Aerosol Drug Management Improvement Team. Respir Med. 2006;100: 1479-1494.

6. Bosnic-Anticevich SZ, Sinha H, So S, and Reddel HK: Metered-dose inhaler technique: The effect of two educational interventions delivered in community pharmacy over time. J Asthma. 2010;47:251-256.

7. Tsang KWT, Lam WK, Ip M, Kou M, Yam L, Lam B, Cheung MT, Lauder IJ, and Kumana CR: Inability of physicians to use metered-dose inhalers. J Asthma. 1997;34: 493-498.

8. Self TH, Arnold LB, Czosnowski LM, Swanson JM, and Swanson H: Inadequate skill of healthcare professionals in using asthma inhalation devices. J Asthma. 2007;44:593598.

9. Kim SH, Kwak HJ, Kim TB, Chang YS, Jeong JW, Kim $\mathrm{CW}$, Yoon HJ, and Jee YK: Inappropriate techniques used by internal medicine residents with three kinds of inhalers (a metered dose inhaler, Diskus, and Turbuhaler): Changes after a single teaching session. J Asthma. 2009;46:944-950.

10. Scarpaci LT, Tsoukleris MG, and McPherson ML: Assessment of hospice nurses' technique in the use of inhalers and nebulizers. J Palliat Med. 2007;10:665-676.

11. The Aerosol Drug Management Improvement Team (ADMIT): Inhalers: dos and don'ts [Internet]. 2010 [last viewed July 14, 2015]. Available at: www.admit-online.info/index .php?id=311\&L=0

12. Everard ML, Devadason SG, Summers QA, and Le Souëf PN: Factors affecting total and "respirable" dose delivered by a salbutamol metered dose inhaler. Thorax. 1995;50: 746-749.

13. Rau JL: Practical problems with aerosol therapy in COPD. Respir Care. 2006;51:158-172.

14. Tzou TZ, Pachuta RR, Coy RB, and Schultz RK: Drug form selection in albuterol-containing metered-dose inhaler formulations and its impact on chemical and physical stability. J Pharm Sci. 1997;86:1352-1357.

15. Michael Y, Snowden MJ, Chowdhry BZ, Ashurst IC, Davies-Cutting CJ, and Riley T: Characterisation of the aggregation behaviour in a salmeterol and fluticasone propionate inhalation aerosol system. Int J Pharm. 2001;221: $165-174$.

16. Jinks $\mathrm{P}$, and Hunt K: Improving suspension MDI dose consistency in patient use by incorporation of a novel semipermeable system component. [Abstract]. Presented at Drug Delivery to the Lungs 17, Edinburgh, UK, November 30December 1, 2006. The Aerosol Society, Portishead, UK; pp. 172-175, 2006.

17. Berlinski A, von Hollen D, Pritchard JN, and Hatley RHM: Prompt firing following shaking of a pMDI delivered via a valved holding chamber is important in achieving the correct dose of Flovent. [Abstract]. Presented at the 20th Congress of the International Society for Aerosols in Medicine, Munich, Germany, May 30-June 3, 2015; J Aerosol Med Pulm Drug Deliv. 28:3;A5.

18. Newman SP: Principles of metered-dose inhaler design. Respir Care. 2005;50:1177-1190.

19. Byron PR: Performance characteristics of pressurized metered dose inhalers in vitro. J Aerosol Med. 1997;10(Suppl 1):S3-S6.

20. Berg E: In vitro properties of pressurized metered dose inhalers with and without spacer devices. J Aerosol Med. 1995;8(Suppl 3):S3-S11.

21. United States Pharmacopeia: Chapter 601: Performance quality tests for aerosols, nasal sprays, metered-dose inhalers, and dry powder inhalers [Internet]. US Pharmacopeial Convention, Rockville, MD, 2006 [last viewed April 12, 2016]. Available at: www.pharmacopeia.cn/v29240/usp29nf24s0_ c601_viewall.html

22. Traini D, Young PM, Rogueda $\mathrm{P}$, and Price $\mathrm{R}$ : In vitro investigation of drug particulates interactions and aerosol performance of pressurised metered dose inhalers. Pharm Res. 2007;24:125-135. 
23. Rubin BK, and Durotoye L: How do patients determine that their metered-dose inhaler is empty? Chest. 2004;126: 1134-1137.

24. O'Callaghan C, Everard M, Smith N, and Denyer J: Feedback during treatment results in increased adherence when using a pressurized metered dose inhaler with valved holding chamber in paediatric patients with asthma. [Abstract]. Presented at the European Respiratory Society Annual Congress 2008, Berlin, Germany, October 4-8, 2008; P3933; Available at: http:///rp.ersnet.org//abstract_print_08/ main_frameset.htm (online only). (Last accessed March 11, 2015).

25. Buhl R: Local oropharyngeal side effects of inhaled corticosteroids in patients with asthma. Allergy. 2006;61:518 526.

26. Roland NJ, Bhalla RK, and Earis J: The local side effects of inhaled corticosteroids: Current understanding and review of the literature. Chest. 2004;126:213-219.
Received on January 5, 2016

in final form, July 22, 2016

Reviewed by: Jolyon Mitchell Denise Conti

Address correspondence to: Ross H.M. Hatley, PhD

Philips Patient Care \& Monitoring Solutions Philips Respironics Respiratory Drug Delivery (UK) Ltd

Chichester Business Park

City Fields Way, Tangmere Chichester PO20 2FT

United Kingdom

E-mail: ross.hatley@philips.com 